\title{
The status of occupational safety among health service providers in hospitals in Tanzania
}

\author{
S.V. MANYELE ${ }^{1 *}$, H.A.M. NGONYANI ${ }^{2}$ and E. ELIAKIMU ${ }^{2}$ \\ ${ }^{1}$ Department of Chemical \& Process Engineering, College of Engineering and Technology, \\ University of Dar es Salaam, P.O. Box 35131, Dar es Salaam, Tanzania \\ ${ }^{2}$ Health Service Inspectorate Unit, Ministry of Health \& Social Welfare, P.O. Box 9083, \\ Dar es Salaam, Tanzania
}

\begin{abstract}
Occupational hazards exist wherever health care is practised. However, there is dearth of information on the status of occupational safety among hospital workers in Tanzania. This study was therefore carried to assess the current status of occupational health and safety (OHS) in Tanzanian hospitals and identify key areas for intervention. Data was collected using a self-administered questionnaire randomly distributed to the health service providers (HSPs) working in 14 district, regional and referral hospitals in Tanzania. A total of 430 HSPs (mean age $=42 \pm 8.8$ years) were involved in the study. Female workers accounted for the majority $(71 \%)$ of the respondents. None of the 430 respondents had received training on OHS as a profession. Most of the supervisors were holders of certificates (54.4\%). Only $42.9 \%$ (6/14) of the hospitals, had at least a supervisor with post-graduate degree. Needlestick injuries accounted for the largest part of the most common accidents (52.9\%); followed by splash of blood from patients (21.7\%); burn injury from chemicals (10.6\%); and slippery floors (5.9\%). There was lack of qualified personnel for OHS in all hospitals. Most of the hazardous activities were carried out by nurses and attendants. Chemicals used in hospitals were mainly antiseptics and disinfectants, which causes skin burns during handling and use. Seminars and workshops were the major source $(\mathrm{N}=429 ; 33 \%)$ of information on OHS. The seven major hazardous activities were injection, cleaning, patient care, bedding, dressing of wounds, medication and surgical operation. The largest proportion of HSPs involved in hazardous activities was found at referral hospitals. The mostly available antiseptics were $70-90 \%$ alcohol, $3 \%$ aqueous iodine, chlorhexidine gluconate and cetrimide. In conclusion, the OHS was observed to be inadequate in most workplaces in Tanzanian hospitals. Special efforts including training, exposure to information and creation of awareness, are recommended for improving occupational health and safety in hospitals in Tanzania.
\end{abstract}

Key words: occupational health, safety, hospital, Tanzania

\section{Introduction}

Occupational hazards exist whenever health care is practised (Gestal, 1987). The greatest risks are usually run by the staff of hospitals and research centres. There are a number of consequences of occupational diseases and injuries among hospital workers. These include economic, physical, and psychological damage to the worker and his family (Gestal, 1987).

The Tanzanian Ministry of Health is striving to identify and bring under control at workplaces all health risks; provide effective measures to protect vulnerable groups at risks; discover and improve workplace situations that may contribute to the existence of health risks; and to educate employers, employees and the community on occupational health and safety (OHS). Employers, including the government, are required to provide information for employees to ensure that current legislative requirements are known, and that relevant, up-to-date information is given regarding all identified hazards and their control (Shilla, 1995; Hryhorczuk et al., 2004).

For all types of hospitals in Tanzania and elsewhere, the healthcare workers' general task involve daily contact with patients, permanent contact with diseases and death, the use of specific techniques or procedures and chemicals which exposes them to several occupational diseases and injuries (Hryhorczuk et al., 2004). The main occupational hazards to which health workers are exposed may be classified as infection, accident, radiation, exposure to noxious chemicals, drug addiction and psychological problems, and assault (Gestal, 1987). In order to safeguard the health service providers (HSPs), the hazards have to be identified, quantified, while the choice of chemicals and their use patterns in hospitals must be adapted to the minimization of risks.

In Tanzania, hospital workers have been observed

\footnotetext{
* Correspondence: Dr. Samwel Manyele; E-mail: svmanyele@yahoo.ca/ smanyele@cpe.udsm.ac.tz
} 
working hazardous environment, and most of them are not aware of the health and safety issues (S. Manyele, unpubl.). This study was conducted to establish the available sources of information on OHS, availability of qualified supervisors in OHS, quantify the hazardous activities in hospitals, and study the distribution of accidents in hospitals. Information on the availability of chemical disinfectants/antiseptics and the frequency of chemical handling in hospitals were also sought. This knowledge was required for improving the training sessions and for recommending necessary interventions.

\section{Materials and Methods}

\section{Study area}

Fourteen hospitals were involved in this study carried out in 2006. They included: Regional hospitals (Iringa, Morogoro, Kagera, Bombo, Lindi, Sumbawanga, person in control of the workplace were employed in data collection. Information collected included qualification of supervisors; common source of information on OHS; hazardous activities; common accidents in workplaces; and chemicals' handling in hospitals. Other data collected include disinfectants and antiseptics use and availability. The interviewee included nurses, medical attendants, nursing officers and medical doctors.

\section{Data analysis}

Data was entered into the computer using a database created in MS Access and MS Excel. The data was then transferred directly into SPSS data editor which provides both data and variable views. The data processing was initiated by invoking the analysis of descriptive statistics and focusing on frequencies. The output contains graphical presentations (histograms and pie charts) as well as tabulated information.

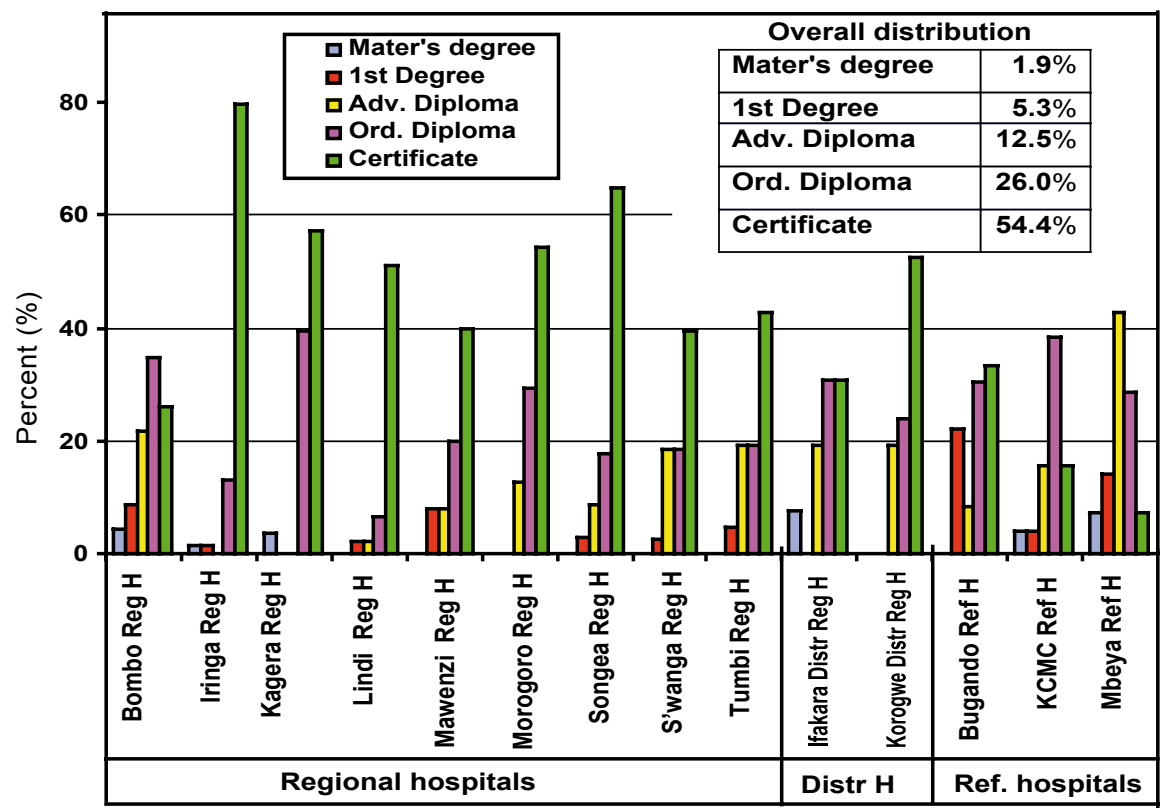

Figure 1: Qualifications of supervisors i 14 hospitals in Tanzania $(N=378)$

Mawenzi, Songea and Tumbi), Referral Hospitals (Bugando Medical Centre (BMC), Kilimanjaro Christian Medical Centre (KCMC) and Mbeya), and district hospitals (St. Francis Hospital in Ifakara and Magunga Hospital in Korogwe). The health facilities and related places of work were sampled at random. Workplaces included wards (medical, surgery and obstetrics and gynaecology), outpatients departments, theatres, laboratories, stores and offices.

\section{Data collection techniques}

Routine inspections with a rapid assessment of the occupational health and safety hazards in the hospitals and personal interview of either the supervisors or the

\section{Results}

A total of 14 health facilities with related workplaces were visited and 430 workers (mean age $=42$ years; $\mathrm{SD}=8.8$ ) were involved in the study. Female workers accounted for the majority (71\%) of the respondents. The distribution of mean age by health facilities shows that the youngest workers were from referral hospitals. None of the 430 respondents had received training on OHS as a profession (Figure 1). Most of the supervisors were holders of certificates $(54.4 \%)$ followed by ordinary diplomas $(26.0 \%)$. In six out of fourteen hospitals $(42.9 \%)$, at least a supervisor with post-graduate degree was available. Most of the supervisors with graduate 
degrees were found in Bugando hospital.

Seminars and workshops were the major source $(\mathrm{N}=$ $429 ; 33 \%$ ) of information on OHS (Table 1). Lack of exposure to information for HSPs was indicated also by least access to internet $(3.7 \%)$ of the information sources and also by fewer number of HSPs with e-mail addresses.

Table 1: Common sources of information on OSH in hospitals

\begin{tabular}{l|c|l|c}
\hline $\begin{array}{l}\text { Source of } \\
\text { information }\end{array}$ & $\begin{array}{l}\text { Per- } \\
\text { cent }\end{array}$ & $\begin{array}{l}\text { Source of } \\
\text { informa- } \\
\text { tion }\end{array}$ & Percent \\
\hline Door-to-door & 1.4 & Posters & 1.2 \\
Internet & 3.7 & Radio & 5.5 \\
Journals and & 5.5 & Seminar/ & 33.0 \\
Guidelines & & Workshop & \\
Leaflets & 4.1 & Supervisor & 19.6 \\
Meeting & 5.5 & Training & 9.7 \\
Newsletters & 6.7 & Television & 4.1 \\
\hline
\end{tabular}

Based on overall analysis, the seven major hazardous activities were found to be injection, cleaning, patient care, bedding, dressing of wounds, medication and operation (Figure 2). During these activities, HSPs were subjected to a wide range of hazards including needlestick injuries, corrosive chemicals, splashes of blood and other body fluids and cuts by sharp objects.
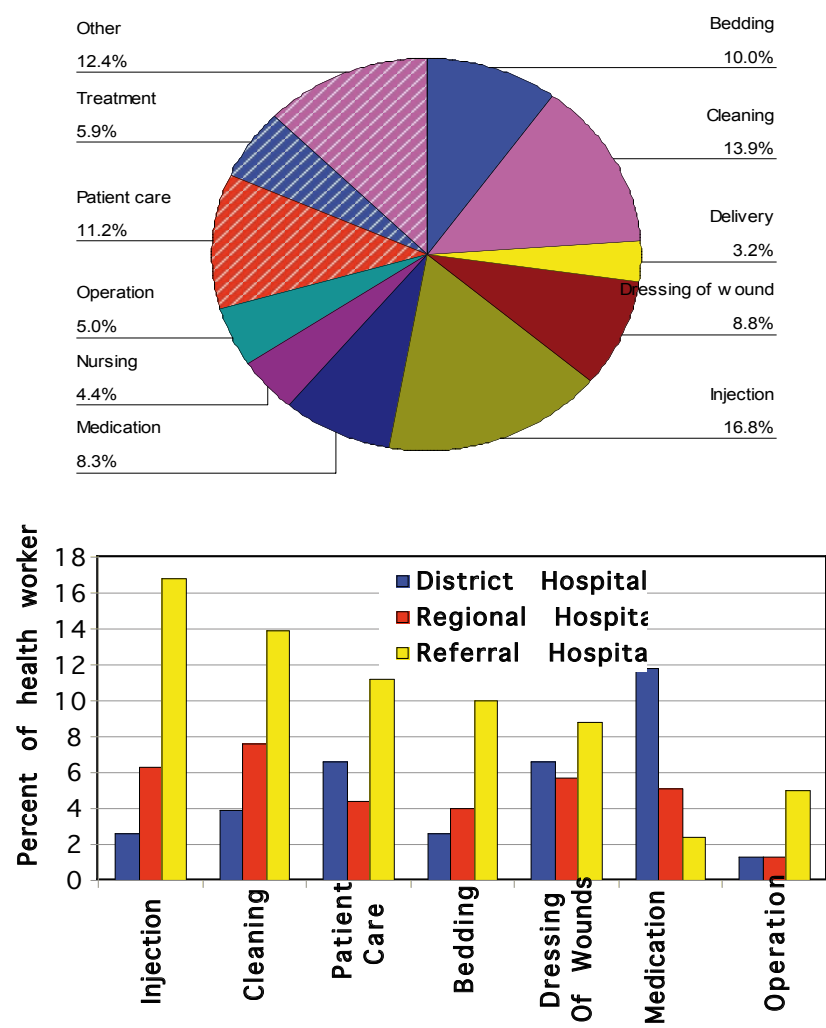

Figure 2: Hazardous activities and specific distribution for different hospital levels in Tanzania

also provided injection services to an appreciable extent, but their major duty was cleaning (Figure 3). It was further observed that bedding and dressing of wounds was again carried out by nurses and medical attendants. Although surgical operation was considered

Most of these activities were carried out by nurses (Figure 2). The identified health risks from workplaces included physical hazards, mechanical hazards, biological and chemical hazards.

The largest proportion of HSPs involved in hazardous activities was found at referral hospitals than in regional or district hospitals. Generally, it was evident (Figure 2) that referral hospitals were more hazardous than regional or district hospitals. Most of the hazardous activities which can lead to infection transmission were carried out by nurses and medical attendants. Most injection services were provided by nurses (nurse midwifery and nursing officers). The medical attendants

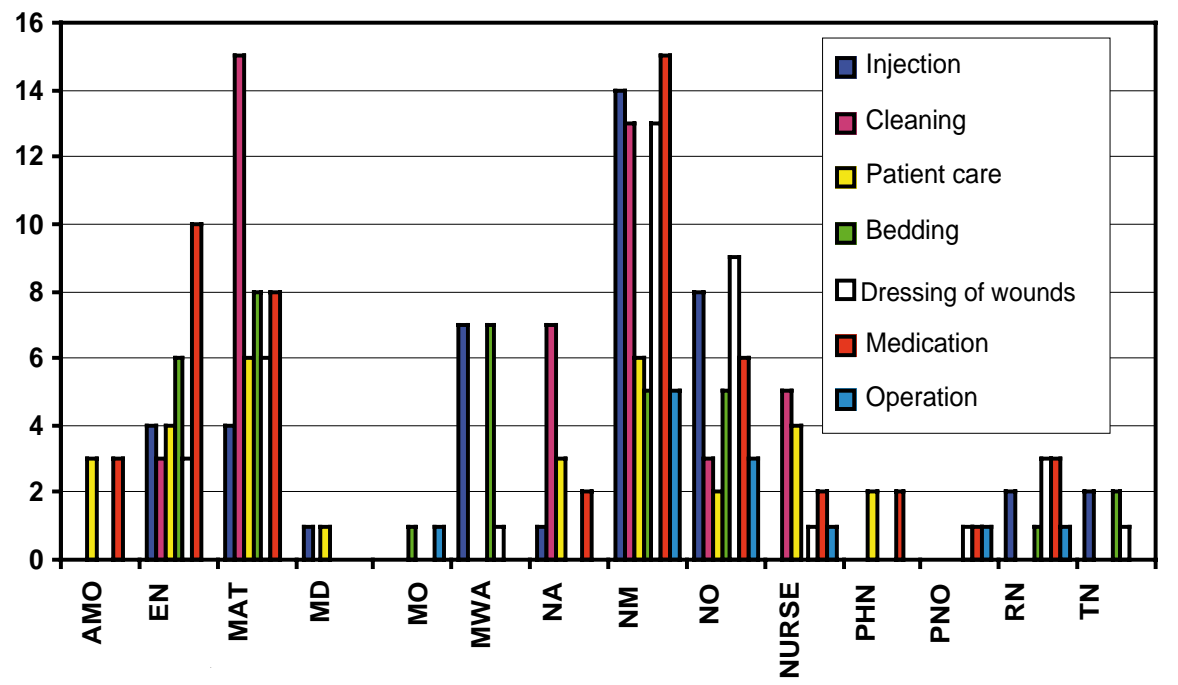

Figure 3: Distribution of hazardous adivities by cadre

Key: $\mathrm{AMO}=$ Assistant Medical Officer, EN = Enrolled Nurse, MAT = Medical Attendant; $\mathrm{MD}=$ Medical Doctor; $\mathrm{MO}=$ Clinical Officer, $\mathrm{MWA}=$ Midwifery attendant, NA $=$ Nurse Attendant, NM = Nurse Midwifery, NO = Nurse Officer, PHN = Public Health Nurse, PNO $=$ Principal Nursing Officer, $\mathrm{RN}=$ Registered Nurse, $\mathrm{TN}=$ Trained nurse 
as a hazardous activity, it was carried out by only a few healthcare providers (Figures 2 and 3).

Five types of hazards/accidents were typical among healthcare workers. These included injuries caused by needle pricks $(52.9 \%)$; splash of blood from patients $(21.7 \%)$; burn injury from chemicals $(10.6 \%)$; and slippery floors $(5.9 \%)$. Many cases of needlestick injuries for HSPs were reported from Iringa, Mawenzi and KCMC hospitals, while the lowest cases were reported in Kagera and Tumbi hospitals. Violence at workplace was rare in the surveyed hospitals, with $3.4 \%$ of the respondents reported to have experiences attacks from patients. This happened particularly in emergency care and psychiatric departments.

All health facilities surveyed had a wide range of disinfectants to choose from. The most prominently used disinfectants were the low-level types. The most accessed high level disinfectant was observed to be chlorine and $5 \%$ cetrimide. The most available intermediate-level disinfectants included methylated spirit, ethanol and providine-iodine. Formaldehyde was least observed because it is used in few workplaces, despite the observation that it was available in all hospitals (Figure

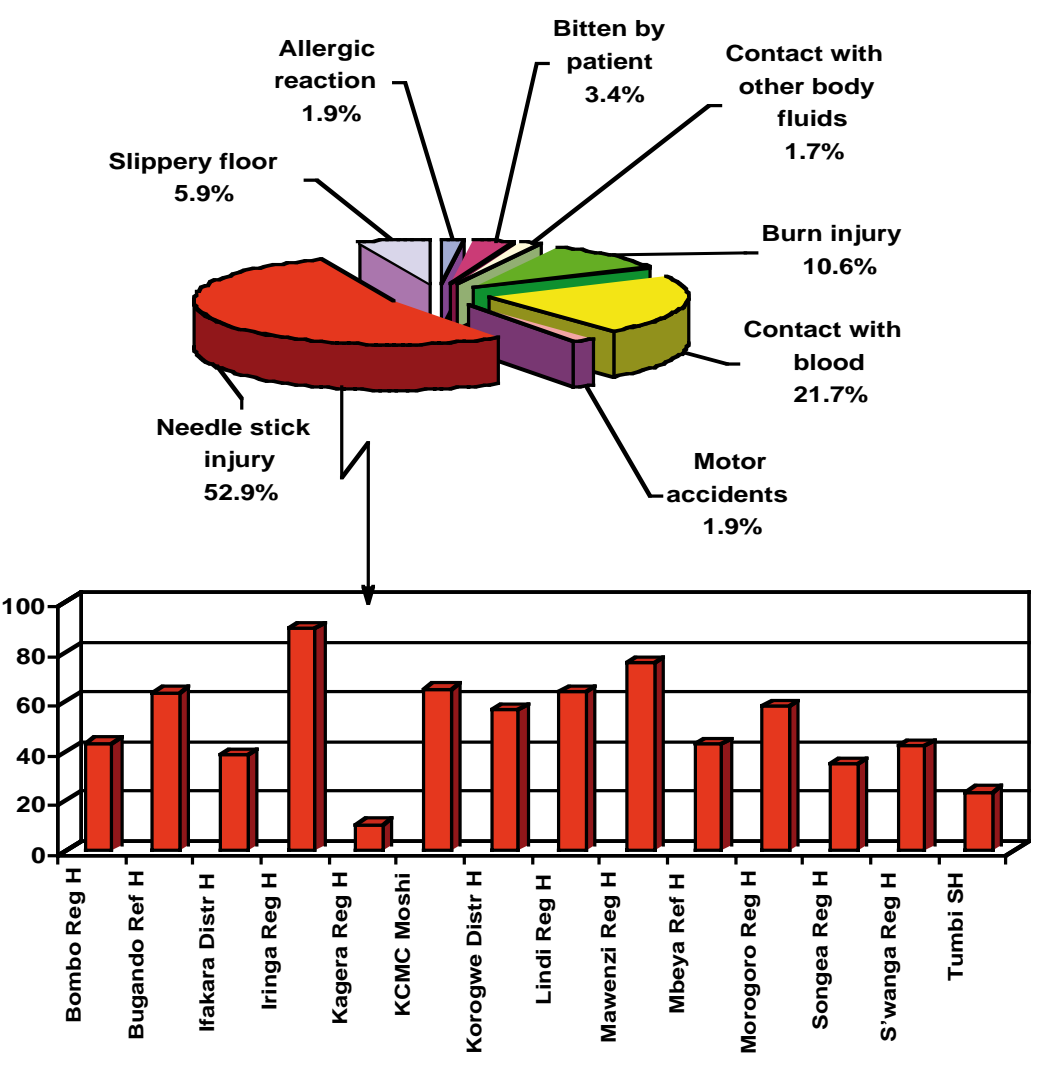

Figure4: Distribution of accidents and needestick injuries in hospital-besed workplaces

5). Moreover, isopropyl (60-70\%) was available in most of the hospitals to a smaller extent.

Table 3: Proportion of use of chemicals in hospitals

\begin{tabular}{lclc}
\hline Name/Group & $\begin{array}{c}\text { Percent of } \\
\text { relative use }\end{array}$ & Name/Group & $\begin{array}{c}\text { Percent of rela- } \\
\text { tive use }\end{array}$ \\
\hline Dettol & $17.7 \%$ & Hydrogen peroxide & $2.2 \%$ \\
Lysol & $17.7 \%$ & Sulphuric acid & $2.2 \%$ \\
Jick & $15.9 \%$ & Methanol & $2.0 \%$ \\
Chlorine & $14.1 \%$ & Formalin & $1.7 \%$ \\
Spirit & $9.2 \%$ & Cetrimide & $1.2 \%$ \\
Savlon & $5.0 \%$ & Hibitane & $0.7 \%$ \\
Iodine & $4.7 \%$ & Phenolics & $0.7 \%$ \\
Fixer/Developer & $3.0 \%$ & Povidine & $0.7 \%$ \\
\hline
\end{tabular}




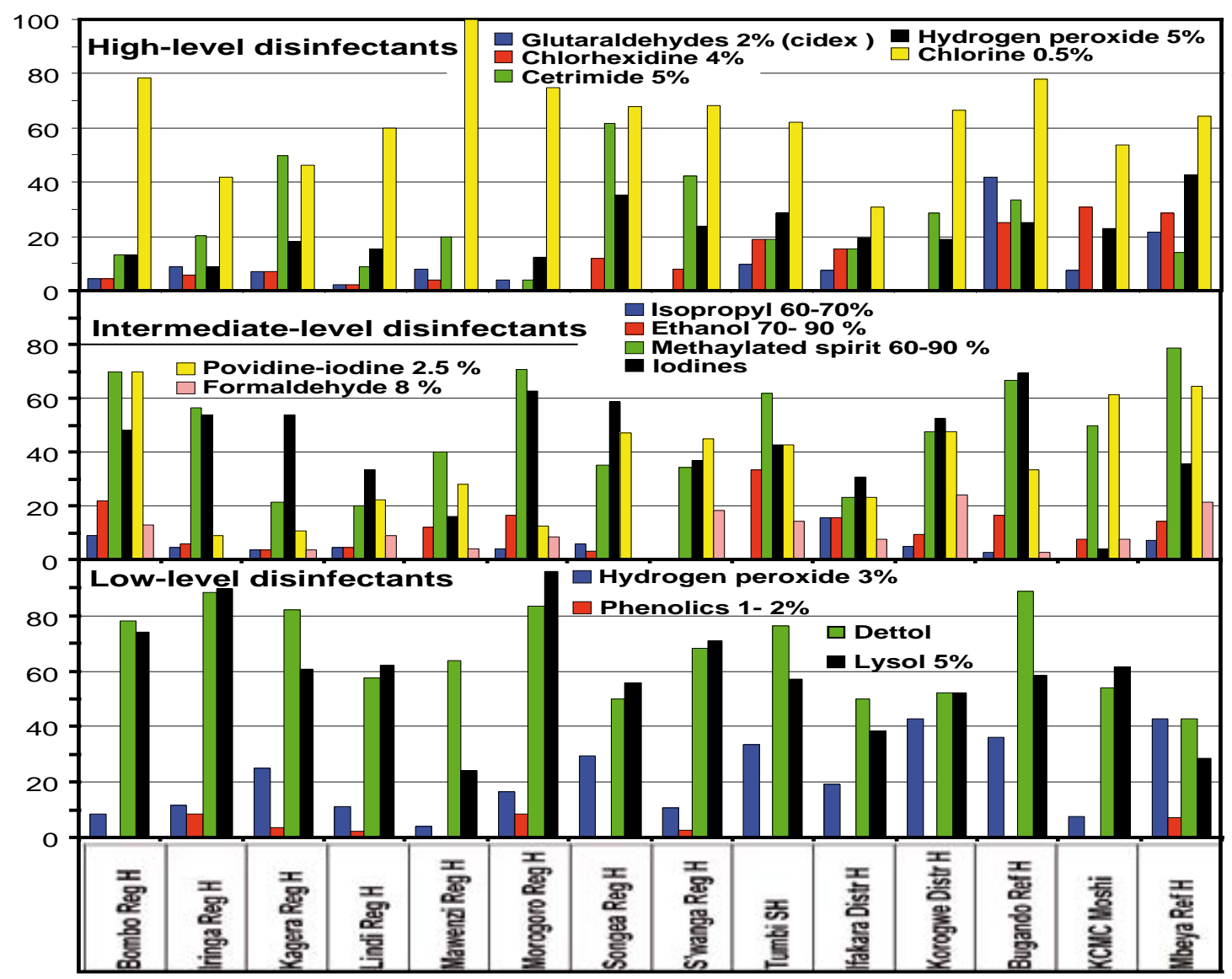

Figure 5: Availability of disinfectants in Tanzanian hospitals (percent health workers with access to disinfectants in workplaces)

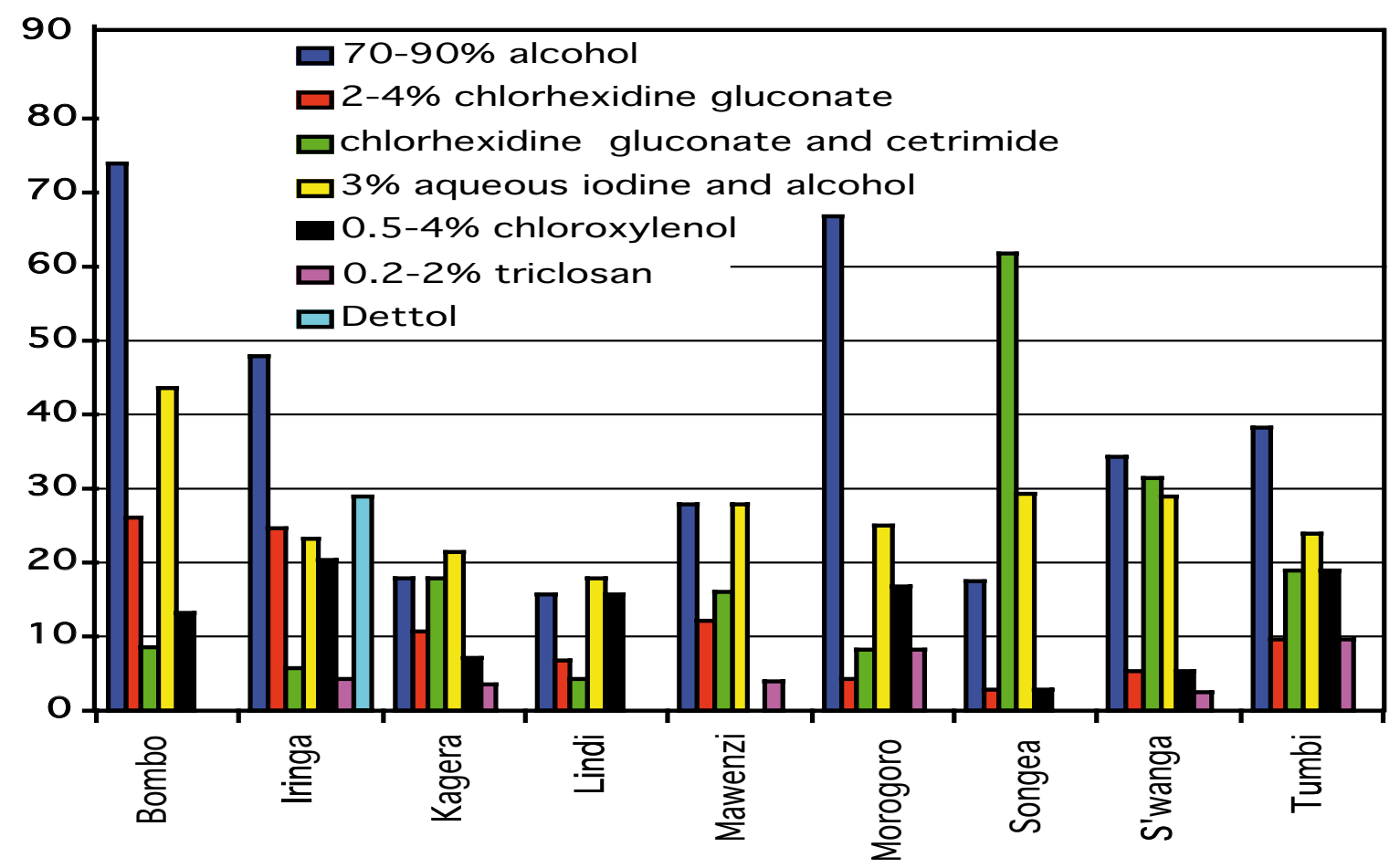

Figure6: Availability of antiseptics in regional hospitals in Tanzania 
A number of chemicals classified in this study as high-, intermediate-, and low-level disinfectants were observed in hospitals (Figure 5). Antiseptics were not equally available in the hospitals, due mainly to procurement problems and problems inherent in the supply chain for the drugs and other supplies in government hospitals. While in other health facilities the HSPs had high access to antiseptics, Kagera, Lindi and Mawenzi hospitals had accessibility to antiseptics below 30\% of HSPs. The mostly available antiseptics in Tanzania were observed to be: $70-90 \%$ alcohol, $3 \%$ aqueous iodine and alcohol, and chlorhexidine gluconate and cetrimide. Dettol was only reported to be available at Iringa hospital. Bombo, Morogoro and Iringa hospitals indicated higher uses of $70-90 \%$ alcohol followed to the similar extent by Tumbi and Sumbawanga Regional Hospitals. Chlorhexidine gluconate and cetrimide were mostly observed in Songea hospital, while Iringa and Sumbawanga hospitals had all antiseptics in use in different workplaces.

\section{Discussion}

Our findings indicate that there is a shortage of health workers trained on occupational health and safety in both levels of hospital in Tanzania. There is lack of qualified personnel for OHS in hospitals and that OHS is given least priority in hospitals. Most of the hazardous activities are carried out by nurses and attendants who comprise the most risky group. Occupational health and safety is a rapidly developing discipline that is continuously being faced with arising challenges from the introduction of new chemicals, new technologies and new work practices (Uusitalo \& Naidoo, 1995). Until recently, the OHS issues have not been given appropriate attention even at the Ministry level. For example, the OHS unit at the Ministry of Health has been manned by one staff until 2000-2003, when two more staff members were recruited. At the moment, the total number of staff at the unit is five. The shortage of trained manpower in OHS can be alleviated by establishment of courses in this area in Tanzania, especially in higher learning institutions. It is evident from this and other studies that little or insufficient attention is given to the OHS problems of the hospital workers (Caillard, 1993; WHO, 1993). Reasons for poor OHS in hospitals can be attributed to the following: the history of OHS, primarily levelled to manufacturing industries and inability of the staff itself to take there matters into account. They give the best of themselves to the patients without any consideration of their own health (Caillard, 1993).

Needle prick injuries were the most common injuries among health care provider in the hospitals. Needle pricks are likely to be the major source of infectious occupational diseases to which the health worker is prone (Gestal, 1987). Of the occupational diseases, hepatitis B and HIV are of greatest importance (Gestal, 1987; Niu, 2000). HIV/AID is currently considered to be the infectious disease giving rise to most anxiety among hospital workers (Geddes, 1986). Injuries due to needlestick or pricks and sharp instruments are the most typical types of occupational accidents, the seriousness of which is due to the risk of infections from contaminated blood (Caillard, 1993). The better way of presenting needlestick injuries is by the number of incidences per nurse per month. A strong cooperation between the heads of the health units, the nurses and all other workers, including the cleaning personnel, in order to attain "zero accident” conditions in all hospital units is recommended (Caillard, 1993).

The identified hazardous activities in hospitals create high chances of acquiring infections for the HSPs. The first task would be to change the mind set of HSPs from service provision at the risk of their own lives to improved self-defence during normal routines. Awareness raising, information flow to and from the HSPs need to be improved.

Medication was identified to be one of the hazardous activities in hospitals, which was highly observed in district hospitals. A great number of drugs used in hospitals are toxic, which can cause allergic dermatosis. Penicillin for example, may also provoke asthma (http:// www.whale.to/drugs/antibiotics_asthma.htm). Of great importance to OHS, are the mutagenic, feratogenic and carcinogenic effects of some cytostatic drugs, especially those with action o the DNA (Caillard, 1993). Beyond the patient safety concerns arising from the necessary therapeutic use of drugs, occupational risks to health care workers handling these drugs in the course of their duties still need to be fully addressed. Chemicals used are mainly antiseptics and disinfectants, which can cause skin burns during handling and use.

Similarly, many chemicals are available in Tanzanian hospitals for use as antiseptics, as revealed in this study. In choosing an antiseptic, the desired characteristics (e.g., absorption and persistence) should be considered along with evidence of a given product's safety and efficacy, its acceptability to staff and most importantly, its cost (Boyce \& Pittet, 2002; Rutala, 1996; Lasron, 1995).

In conclusion, the OHS was observed to be inadequate in most workplaces in Tanzanian hospitals. Special efforts including training, exposure to informa- 
tion and creation of awareness, are recommended for improving occupational health and safety in hospitals in Tanzania. It is important that all hospital workers are taught and trained in safety measures. There is lack of formal training on OHS in the country. Special efforts including training, exposure to information, awareness rising, are recommended for improving OHS in hospitals. It is the responsibility of the employer to provide healthy and safe workplaces for all HSPs in line with the requirements of the relevant national and international laws and regulations, and to organize the work in such a way that the exposure of HSPs to hazardous factors and risks at work are eliminated or minimized, and their safety and health is protected. It is worthy mentioning that the Ministry of Health faces a critical lack of occupational health and safety personnel in hospitals. This highlights the need to do everything possible to look for alternatives and available resources to tackle this problem. The first thing is to train the supervisors at different levels in $\mathrm{OSH}$ so as to equip them with necessary skills to identify and eliminate occupational hazards in hospitals.

\section{Acknowledgements}

This study was conducted in collaboration with the Environmental and Occupational Health and Safety Centre, in Dar es Salaam. The assistance from the IPC/IS National Facilitators during data collection exercise is highly acknowledged. The authors are also grateful to the Occupational Health Unit of the Ministry of Health and Social Welfare, Tanzania, for their contribution.

Received 5 October 2007

Revised 17 April 2008

Accepted 24 June 2008

\section{References}

Boyce, J.M. \& Pittet, D. (2002) Guidelines for Hand Hygiene in Healthcare Settings: Recommendations of the Healthcare Infection Prevention Control Practices Advisory Committee and the HICPAC/SHSA/APIC/IDSA hand Hygiene Task Force. Infection Control and Hospital Epidemiology 23, S3-340.

Caillard, J.F. (1993) General Aspects of Occupational Health for Healthcare Workers. African Newsletter on Occupational Health and Safety. Proceedings of PACIH'92, 3, $78-85$.

Geddes, A.M. (1986) Risk of AIDS to health care workers. British Medical Journal 292, 711-712.

Gestal, J.J. (1987) Occupational hazards in hospitals: risk of infection. British Journal of Industrial Medicine 44, 435-442.

Hryhorczuk, D., Nickels, L., Forst, L. \& Zanoni, J. (2004) Online training resources in occupational safety and health. African Newsletter on Occupational Health and Safety 14, 19 - 22.

Larson, E.L. (1995) APIC guidelines for handwashing and hand antisepsis in healthcare settings. American Journal of Infection Control 23, 251-269.

Niu, S. (2000) Occupational safety and health in the health care sector. African Newsletter on Occupational Health and Safety 10, 56 - 59.

Rutala, W.A. (1996) APIC guidelines for selection and use of disinfectants. American Journal of Infection Control 24, 313-342.

Shilla, C.P.N. (1995) The power of information. African Newsletter on Occupational Health and Safety 5, 3.

Uusitalo, T. \& Naidoo, R. (1995) Accessing occupational health and safety Information through the Internet. African Newsletter on Occupational Health and Safety 5, 6-8.

WHO (1993) World Health Organization: Occupational Hazards in Hospitals. EURO Reports and Studies, Copenhagen, p. 68. 